\title{
A Feel for the Game: Exploring Gaming 'Experience' Through the Case of Sports-Themed Video Games
}

\author{
Garry Crawford, Daniel Muriel, \& Steven Conway.
}

\begin{abstract}
Video gaming is often understood and narrated as an 'experience', and we would suggest that this is particularly notable with sports-themed video games. However, we would argue that how the game experience is curated and consumed, and how this relates to wider social process and forces, is rarely given any detailed consideration within the existing game research literature. Hence, this paper explores how game experiences can be understood and articulated around four key themes. First, we begin with the argument that video games connect with, but also lead, a wider social trend: understanding social reality as a set of designed experiences. The real is progressively becoming a repository of technologically mediated experiences, and the logic of video games is anticipating this process. Second, we suggest video games are translations of phenomenological worlds: when successful, key aspects of the meaning of things remains similar even as one moves between spaces, domains, mediums, and platforms. Developers often seek to bring others' experiences into a game environment, such as translating the geography and mechanisms of sporting locations and competitions into a game environment. Thirdly, following this translation of meaning across domains, gamers often narrate their encounters with video games as they would any other experience, such as winning the Champions League in Football Manager becomes recounted by gamers like any other achievement. Fourth, video games are interactive and explicit bodily experiences since they must be enacted in order to exist.
\end{abstract}

Keywords: experience, phenomenology, interactivity, sports, theming, video games, hyperreality, mediations

\section{Introduction}

This paper proposes 'experience' as a foundational concept and tool for understanding video games and video gameplay, and in particular, here employs 'sports-themed video games' as a key exemplar to explore the usefulness of this focus. It is not our argument that we are the first to discuss video games from the perspective of experience, or emphasise the importance of the gaming experience. As we shall highlight, many academics, journalists, bloggers, players, and others have often talked and written about video games as experiences. In this sense then, video games are usually understood from a phenomenological perspective; as phenomenology is the study of experience, and games are, more so than other media, explicit in their 
embodiment and insistence upon extranoematic (outside of thought) enactment, regardless of how trivial or illusive this agency ultimately is.

Indeed, this is our point. Video games and gameplay are often articulated as, or related to, experience, but rarely is what this means or how it helps us better understand video games and gaming explored in any detail. Hence, to this end, this paper seeks to set out experience as a model and tool for understanding video games and video gameplay, and in particular, uses sports-themed video games as an illustration and application of this model to a case, which we would suggest provides a key example of the importance and prominence of experience in video gaming discourses.

Hence, this paper explores how the gaming experience can be articulated around four key themes. First, we suggest that video games connect with, but also lead, a wider social trend: understanding social reality as a set of designed experiences. Second, we consider video games as translations of meaning across domains. Developers often seek to bring others' experiences into a game environment, such as translating the geography and mechanisms of sporting locations and competitions into a game environment. Third and consequently, gamers often narrate their encounters with video games as they would any other experience, such as going on a trip. Fourth, the specific bodily insistencies of the digital game medium aid the generation of this meaning and narration as 'sports-themed'. Before unpacking these four lenses, we (briefly) explore the notion of experience and re-visit our case for considering and referring to this genre of games not simply as 'sports video games', but rather 'sports-themed video games'; a discussion which we suggest has particular relevance to the subject under consideration here. Finally, we highlight that this paper makes three significant contributions to knowledge. First, it defines and operationalizes a key concept that is very often used, but rarely defined, by many games designers and academics. Second, this paper frames discussions of experience within a wider social and economic context and suggests that video games provide a key example of how increasingly social reality is being framed and sold as designed experiences. Third, we also suggest that experience offers some common ground in the, now somewhat dated but still relevant, 'narratology versus ludology' debate.

\section{Exploring Experience in Video Games}

The categorization of video games as a form of experience is, to some extent, identified and explored by many game scholars. Katie Salen and Eric Zimmerman's 
Rules of Play is, for instance, mainly about designing experiences for players by 'understanding how a game's formal system transforms into an experiential one' (Salen and Zimmerman, 2004: 316). Experience is a key notion in their definition of video games; which stresses that a game designer does not create technology but rather 'an experience' (Salen and Zimmerman, 2004: 87). Salen and Zimmerman are therefore directly equating video games to experiences; assuming that playing a videogame is to have a particular experience. Peter Bayliss (2008) is unequivocal on this point, emphasising that gameplay 'is understood here as an embodied experience that emerges from the activity of play, rather than a property of the game being played'. James Ash's (2013) work further develops Heidegger's notion of attunement, describing the digital game medium's various methods of attuning one's corporeality and affectivity to the game environment.

Furthermore, Ian Bogost (2007: 35) suggests that the procedural representation of video games is, though not identical to what we could consider an 'actual experience', capable of simulating 'real or imagined physical and cultural processes'. Moreover, the interactivity of video games, he suggests, could locate them towards the very top of a scale of experience vividness. Drawing on the psychologist Charles Hill (2004) and his continuum of vividness, which sets-out a scale that ranges from the least vivid information (statistics) to the most vivid one (actual experience), Bogost (2007: 34-35) situates video games right under actual experience and above moving images with sound (which occupies the second spot in Hill's list). Although, we would suggest defining actual experience is highly problematic, not to mention the very idea of vividness, Bogost seems to reach a plausible conclusion: that video games are meaningful experiences.

\section{Experience and Sports-themed Video Games}

Frequently, different actors that belong to video game culture, including academics, journalists, bloggers, people working in the industry, and gamers themselves, express that when they are playing, studying, writing about, and designing video games, they are doing it, mostly, in terms of experience. As highlighted earlier, video games are not just played, they are experienced: the play activity is experience, and furthermore this experience can, and often is, linked across what are otherwise separate domains. This can be notably seen in (what we term) 'sports-themed video games'. For example, Adi (2011) on the sportskeeda.com website wrote of football 
management themed games, such as Football Manager, that 'manager games, while nowhere as popular as either Electronic Arts' FIFA or Konami's PES [Pro Evolution Soccer] series, once played and experienced, can be incredibly addictive'. Also, Abe Stein (n.d.) wrote on Kill Screen that 'at the heart of the whole Madden experience is the simulation of the game on the field'. Implicit in both quotes is an assumed commonality of experience; in the former across genres, in the latter across mediums. Hence, this repeated articulation of video games as 'experience' is the focus of this paper, and also why here, following AUTHOR1 (2015), we suggest the use and particular relevance of the term 'sports-themed video games' as an exploratory probe.

AUTHOR1 (2015) proposes the use of the term 'sports-themed' for three key reasons. First, referring to this genre of videogame as sports video games, implies, either explicitly or implicitly, a direct and simple link between sport and its representation in videogame format. That is to say, they are typically understood as either directly simulating a sport, or as Ian Bogost (2013) has argued, as sports in themselves. This argument, and in particular the work of Bogost here, we find very problematic. Bogost (2013: 53) presents a distinctly weak case for games as sports, suggesting simply that 'just about anything can be taken seriously as...a sport', and hence, his line of argument follows, then why not sports video games? Yet, as Bernard Suits (2005) famously summarised in his illustration of the 'lusory attitude', 'seriousness' has nothing to do with sport, but everything to do with play. One can run a 400-metre race and not take it seriously, in which case one is not playing (instead 'trifling'), but, as long as one follows the rules, who could object that the runner is in fact partaking in the sport of 400-metre racing?

The complex relationship between sport and video games, and whether video games should be considered as a sport, has been explored or at least touched on by numerous authors, including (but not limited to) Hutchins (2008), Taylor (2009), Taylor (2012), and Witkowski (2012), and, certainly, this is a debate that has remained unresolved for decades. Ultimately, it is a debate that remains unresolved since a universally agreed upon definition of what a sport is itself, remains elusive. It is therefore somewhat difficult to seek to define video games as a sport, when there is little agreement over what a sport is.

This lead to, and relates to the second key issue here, which Bogost (2013) himself acknowledges, in that, 'sports video games' (as Bogost, and most others, refer 
to them) are not an holistic simulation of a sport, no matter how much they lean upon the rhetoric of 'the real' in their marketing. Most notably, sports video games do not, and currently cannot, replicate all aspects of a sport, such as, its economics, infrastructure, dietetics, training regimes, et cetera. As Ernest Adams (2010), argues, rather, this genre of video game tends to focus only on one, or a small number, of the aspects and mechanisms of a sport. As Adams (2010: 482) writes 'a sports [video]game simulates some aspect of a...sport'.

Third, not only do 'sports video games' attempt re-presentation of certain aspects of a sport, they are still always at the mercy of the digital game medium's essence, namely the necessity of calculation (AUTHOR 3, 2016). For example, games like, Pro Evolution Soccer, FIFA and Football Manager (as do most games of this nature) place numerical values on the unquantifiable, such as passing and shooting technique, while the EA Sports' Street series of games, such as NBA Street and NFL Street, utilise exaggerated game-world physics and caricatures of the athletes (AUTHOR1, 2015).

These, and others arguments, explored by AUTHOR1 (2015), lead to the assertion that what are frequently termed 'sports video games', are best understood, not as sports in themselves or as a simulation of a sport, but rather as sports 'themed'; draped in meaning pertinent to sport. This, he argues, is useful as it allows us to explore the relationship between in-game and out-of-game spaces, and how video games (more generally) often seek to draw on, but also extend the possibilities of, other social spaces. As Espen Aarseth (2007: 163) writes, 'computer games are both representations of space (a formal system of relations) and representational spaces (symbolic imagery with a primarily aesthetic purpose)'.

The structure and basis of a game's code is often similar across many different games; indeed an enormous number of digital games are built within just very few cross-platform game engines, such as Unity, Unreal Engine, or CryEngine. Often then for sports-themed video games, what gives each its (perceived) 'uniqueness' is the theme laid upon that structure. For example, in the case of a sports-themed videogame like FIFA, it is the use of official team names, colours and rosters, the various competition's structures and insignia, and the modelling of player appearance and stadium settings, which (re)creates the meaning of professional, international football. Quite simply, to use AUTHOR3's (2015) SOC (Social/Operative/Character) model, 
whilst sports-themed video games can never fully imitate the 'Social World'1 of sport, they do indeed leverage aspects of its Operative and Character world. That is to say, the Social world (class, gender, race, culture, and so forth) is largely unaddressed by these games in any real depth. The Operative World is mostly not emulated: whilst rules are perfectly imitated, the physical kicking of a football, the feel of grass, a competitor's elbow in your ribs is not. ${ }^{2}$ Yet its Character World, the meaning the world of sport holds for the player, fan and larger culture (identification with a team, its colours, emblem, history, stadium, roster and so on), is very well leveraged by the sports-media complex. The ultimate goal is to translate engagement and understanding from one domain, such as experiencing the sporting event as a fan or player, into another, the experiencing of a video game as emulating this same meaning. Hence, as we shall argue below, these kinds of video games do not therefore offer a simulation of the sport, but rather a translation of aspects of the meaning of sport into a videogame format. They are not a sports simulation, as many have often described them, but rather should be seen as sports-themed experiences.

How we can consider video games as experiences is set out and explored below. In particular, as stated above, there are at least four relevant reasons why video games could be associated to the notion of experience, which in this paper we will seek to elaborate and apply to the case of sport-themed video games.

\section{Social reality as a set of designed experiences}

First then, we turn to the idea of video games as an example of a wider social process; where by social reality is increasingly being encountered (and sold) as a set of designed and curated experiences. We live in an epoch that, via institutions such as the culture industries, emphasise the production, distribution and consumption of designed experiences. The economists Pine and Gilmore (2011) consider that from the end of the $20^{\text {th }}$ Century there started to emerge a new kind of economy, one based on the production of experience. Not only do they think this is an emerging reality, they also deem it economically necessary; as the paradigm centred on the production of goods

\footnotetext{
1 'Worlds' here should be understood in the phenomenological sense as domains within which certain meanings pertain. For example, the 'world' of coffee, the 'world' of art, the 'world' of sport: in each, different meanings are attached to the same material reality. For example, a cup in a coffee house has a different meaning to a cup in an art gallery.

${ }^{2}$ Even motion-based or motion-sensing technologies, such as the Nintendo Wii or Microsoft Kinect, will only attempt simulation of one or two aspects of the Operative World, such as kicking, and even then one is kicking thin air.
} 
and services is no longer viable in late capitalism. This the authors call the experience economy, which is based on offering staged experiences to customers instead of goods (Industrial Economy) or services (Service Economy): 'in a world saturated with largely undifferentiated goods and services the greatest opportunity for value creation resides in staging experience' (Pine and Gilmore, 2011: ix). For Pine and Gilmore this 'value creation' is mutually beneficial for both the producer and consumer; as the latter gets a more enjoyable, prolonged, and engaging encounter, while the former profits from being able to sell something customers seem to want and enjoy.

According to the authors, the beginnings of this rise in the production and sale of experiences can be traced back to Walt Disney's theme park Disneyland; however, the rapid development of new technologies since has encouraged 'whole new genres of experience, such as video games, online games, motion-based attractions, 3-D movies, virtual worlds, and augmented reality' (Pine and Gilmore, 2011: 4). Building upon this, we would suggest that video games appear to be the pinnacle of this new codification of social reality as designed experiences. Andrew Grove (one of the founders of Intel and considered to be an early prominent guru of Silicon Valley) anticipated the explosion of technology-enabled offerings in the mid-1990s when at the COMDEX computer show he stated: 'We need to look at our business as more than simply the building and selling of personal computers [that is, goods]. Our business is the delivery of information [that is, services] and lifelike interactive experiences' (Pine and Gilmore, 2011: 4). It is not surprising then to see how traditional service industries are also becoming overtly experiential in how they frame their suite of products.

In particular, we would suggest video games are a key exemplar of this trend, which is pointing towards a society that was anticipated by postmodern theorists, such as Baudrillard (1994). Here, Baudrillard explores the notion of the hyperreal, which involves a rhetorical embedding of 'the real' within the very affordances of the medium. For example, in television High Definition (and now Ultra High Definition) is discursively framed as bringing one closer to the 'reality' of whatever is depicted; this is also true of associated technology such as High Dynamic Range (HDR) colour and high-fidelity sound. This then, is what video games and in particular sport-themed video games offer; a perceived 'real' experience, one that claims to as closely as possible simulate the 'real sporting world'. As games journalist George Osborn (2016) wrote in his review of Football Manager 2017 on The Guardian online: 'FM 2017 offers the 
best experience of pretending to be a football manager there's ever been'.

Yet perhaps Osborn is not bold enough in his assessment, as in many ways the digital game experience is even more emphatic and spectacular than the reality. In place of ambiguity (of your squad's abilities, mentality, coaching staff capacity), we have the transparency and reliability of the digital game's numbers and statistics; in place of scarcity (of resources, matches, interactions, ability to control and influence your team), we have abundance (endless seasons, thousands of interactions, extreme control over the club and squad); as opposed to banality and boredom, we encounter the intensity of the digital game medium, to provide immediate interaction, to offer instant participation, to insist the player is important and that participation matters ('you've won the Premiership!'). The experience is condensed, multiplied, and reconfigured for spectacle: designed as, and in service to, a hyperreal that never has, nor could, exist in the day-to-day management of a sport team.

Yet this strategy is so convincing that even professional athletes start to buy into the rhetoric that the games equate to, and in some ways provide, superior access to the 'real experience'. For example, in 2017 when the New York Giants' defensive tackle player Damon Harrison was asked by the New York Daily News about his lack of playoff experience, he replied that in fact he had plenty of experience, as he had already won the Super Bowl playing Madden NFL. As Harrison stated in the interview: 'I just won a Super Bowl last night on Madden with the Green Bay Packers. I go to the playoffs every year on Madden, so I have some playoff experience if guys want to talk to me and lean on me' (Popper, 2017). This could be dismissed as the lighthearted quip it probably was, but there is a long and well-established history of sports managers and players looking to sports-themed video games for knowledge and experience. For example, in 2014 the makers of the Football Manager series Sports Interactive signed a deal with Prozone Recruiter, which would allow football club managers and scouts to use their database of over 80,000 players to search for new talent and signings (Stuart, 2014).

Certainly, it can be argued that many video games present key examples of a 'staged authenticity' (MacCannell, 1973). As Stallabrass (1996: 102) writes, 'many [video]games take the form of staged, tourist exploration... [but] as with the exploitation of "heritage" themes...they are collected, combined and packaged as entertainment, inevitably with a strong flavour of pastiche'. The comparison that Stallbrass makes 
between video games and the areas of tourism and cultural heritage is undoubtedly very relevant, as those are fields that have been particularly permeated by experience staging (see AUTHOR2, 2017). Hence, this is an important point made by Stallabrass, and one that returns to the ideas of Baudrillard (1994) on hyperreality. Though sports-themed video games discursively frame the experience provided as 'authentic', they are innately pastiche through their infusion of digital software (and as we shall argue later, televisual) conventions. As AUTHOR3 has phrased it, such experiences are not simply 'mirrors' of sport, but carnival mirrors (2013): misshaping, exaggerating, obfuscating in their translation.

Also, as noted, inauthenticity is inescapable: the experiencing of these environments through the mediation of light-emitting diodes (LEDs) upon a television screen; through the haptic feedback of a game controller; through the voice activation of the Microsoft Kinect; all highlight the inauthenticity of the experience. This is perhaps why the discursive framing of the various representational tropes in sports-themed video games as 'reality' is so pervasive; to divert from the essential ersatz complex of the medium and its developers. Hence, AUTHOR1 (2015), drawing on the work of Feifer (1985), suggests that the gamer is possibly best conceived as a 'post-tourist'. That is to say, for Feifer, the post-tourist accepts elements of staged inauthenticity, such as the Venetian gondolas in Las Vegas, as these add to their tourist experience. As Pine and Gilmore remind their readers, 'staging experiences is not about entertaining customers; it's about engaging them' (Pine and Gilmore, 2011: 45). It is not uncommon to read in design manuals that a video game designer should know how to translate 'the formal intricacies of the rules into an engaging experience of play' (Salen and Zimmerman, 2004: 330). This is then what video games are supposed to be: designed engaging experiences that may blur the boundaries between various phenomenological worlds (the worlds of sport, video games, and television shows) and technologies (television, computing, software and 'big data'). Yet they must still give the sense of enacting and embodying an experience of playing or otherwise engaging in sport: the experience of an experience.

\section{Translating experiences}

Our second theme is the idea of video games as translated experiences. One of the most widely spread ideas that traverse video game culture is that video games allow players to access experiences that they would not have otherwise; as Shaw (2014: 87) 
noted during her research on marginalized gaming cultures: 'For many interviewees, connecting to media characters was about experiences more than anything else'. For example, sports-themed video games have often been marketed on the basis of their ability to simulate the sports-experience. FIFA 06 was promoted with the tagline 'experience football as it was intended', and Madden NFL as 'the most complete football experience'4. The official website of Football Manager 2017 highlights its 'enhanced match experience' (SI Games, 2017). The emphasis here, as with many other sports-themed video games, is about providing 'authentic', meaningful gaming experiences; as guidingtech.com writes of the mobile version of Football Manager, this is 'one of the best football manager experiences's.

The videogame medium claims to push players into the shoes (or boots) of others, allowing them to experience the world from their perspective. Of course, players are not experiencing what others feel or what it means to be in a specific situation. As we have articulated, the medium can only ever achieve pastiche, not even close to the experience itself, but ipso facto in its deployment of pastiche it refers to other realities. This degree of connection varies: players might identify or not with the characters and the situation, or they might connect at an emotional or cognitive level (or possibly both). The important thing is that the reference is established and, in doing so, one is provoked to engage with a familiar sense of meaning, such as the meaning of the world of sport, in a novel way.

According to Salen and Zimmerman (2004: 316), creating great experiences for players 'requires understanding how a game's formal system transforms into an experiential one'. Therefore, once it is possible to establish, at least hypothetically, that video games are able to offer diverse experiences to players, the question arises: How do they do it? How do they convey these various and rich experiences to those who are going to engage with them? The answer to these questions relies on the process of translation that takes the Character (AUTHOR3 2015) world of a specific experience, and translates its germane attributes into the digital game. Which is to say, in the moment of translation for sports-themed video games, the process can ignore the Social (gender, class, race, etc.) world which the live experience inhabits; it can discard much

\footnotetext{
${ }^{3}$ https://fifa-06.en.softonic.com/

${ }^{4}$ https://forums.ea.com/en/discussion/85947/madden-nfl-17-the-most-complete-football-experience

${ }^{5} \mathrm{http} / / / \mathrm{www}$.guidingtech.com/65092/football-manager-games-android/
} 
of the Operative (for example, it can simulate the offside rule but not the physical technique to perform the rabona ${ }^{6}$ ); yet the Character world of sport must remain intact.

Even if the experience the developers are trying to reproduce in a videogame is not directly known to them, they overcome this limitation by feeding on other's experiences. Within a heavily mediatised environment where sensibility and comprehension of phenomena are heavily informed by technological mediation, this is a supremely accessible and effective strategy, as outlined by AUTHOR3's (2013) concept of 'reludification' (building upon Bolter \& Grusin's 'remediation' (1999)). In this manner games developers are curators of experiences across various media; using them as raw material for their own design. Video games consist of a transposition of experience that transforms, mediates, and adapts, with the aim of creating a new experience of familiar content: something accessible, comprehensible, and meaningful.

Video games are, in this sense, experiences of experiences. For example, in relation to sports-themed video games, Abe Stein (2013) highlights how the college football-themed game NCAA Football 06 uses the voice and a digital model of the television commentator Lee Corso. This, Stein (2013:124) suggests, offers one example of 'the remediation of the televisual, incorporating a familiar element of the old medium into the new'. Stein equates the video game experience to that of the television sports fan. Moreover, Stein cites one of the 3D artist and developers of NCAA Football 06, Kyle Wolfe, who highlights how they 'worked closely with the footage from the ESPN [a sports television network] broadcast to design 3D elements that matched the feel and experience of the televised sport coverage' (Stein, 2013: 127). Hence, sport-themed video games most commonly seek to draw on and imitate the televisual spectatorship of sport, making them the experience of the experience of watching the game. Yet this is not just watching, but also being able to guide the actions taking place - a very important difference, and one we will return in a moment.

\section{Narrating experiences}

Third, we turn to the idea of video games as a narrated experience. Chatman (1978: 19) defines a narrative as consisting of two interlinked elements: a story (histoire) — which is the content, the chain of events, characters and settings — and also a discourse (discours) — which refers to the meaning, its expression, and how the

\footnotetext{
${ }^{6}$ Striking the ball by wrapping the kicking foot behind the standing leg.
} 
content is communicated. That is to say, a narrative is a text that tells a story (Kücklich, 2006), but the understanding of this story comes not just from its contents, but also its context, form, expression, and associated meanings.

It was Janet Murray (1997) who famously argued that new computer technologies, such as video games, can extend the possibilities of storytelling; and in doing so gave birth to what would become known as a 'narratological approach' to video game analysis. In particular, Murray argues that computer technologies allow and extend the pleasures of immersion, transformation, and agency. Here, agency relates to making meaningful decisions and seeing the result of these choices; transformation refers to computer technologies' ability to create new environments and roles; while immersion relates to the feeling of being submerged in a different reality. In particular, Murray is very clear that for her computer technologies offer a 'narrative experience' (Murray, 1997: 132). For example, in her elaboration of immersion, she writes: 'the experience of being transported to an elaborate place is pleasurable in itself...We refer to this experience as immersion' (Murray, 1997: 98).

However, Jesper Juul (2001), in his now much cited critique of a narratological approach to the study of video games, suggests that not all video games tell a story. Here, Juul argues that narratives are traditionally located within at least two, possibly three, 'kinds of time'. First, there is the story time, which refers to when the events taking place are set. Second, there is a discourse time, which denotes the time of the telling of the events. For example, a story teller will recount a story to an audience at a particular time, but that story will be set at some other time. Even if this involves recounting something that has just happened, it is still being retold at a different time. Juul then suggests that there is possibly a third kind of time, which is particularly relevant to contemporary narratives, which is the reading or viewing time; often narratives are recorded in media, such as in a film or a book, which are then at a later time viewed by an audience.

Juul, using the example of the first person-shooter Doom II, illustrates how this model of traditional narrative is problematic when applied directly to video games. Here, Juul argues that it is 'hard to find a distance between story time, narrative time, and reading/viewing time', for, as Juul argues, in an interactive videogame like Doom II all of these occur almost simultaneously; 'the story time as synchronous with narrative time and reading/viewing time' (emphasis in original). Hence, Juul argues that 'this 
means that you cannot have interactivity and narration at the same time' (emphasis in original).

Though Juul does not use the example of sports-themed video games, one can easily see how his argument could also apply here. Traditionally, sports-themed video games do not, and in many cases still do not, have an explicit narrative structure. When the player plays a football action or management games, for example, they may perhaps create their own internal narrative, such as their team overcoming great odds to win a famous victory, but this is still happening synchronously; this is a narrative they are helping create in real time, which only exists as an archetypal schematic they then fill in with unique details.

Several authors such as Carr (2006), Albrechtslund (2010), and AUTHOR1 (2012) have argued that Juul marginalizes both the role of the gamer in creating narratives, and how they may recount these to others. It is important to note that Juul does not overlook the narrative possibilities of gamers retelling their gaming experience; as he clearly states in his conclusion to this paper, 'the player can tell stories of a game session'. However, it is certainly the case that he pays little attention to this, and does not see this as any justification for employing narrative analysis in video game research. For Juul, it is still the act of play, rather than how the gamer constructs or retells a game narrative, which matters.

It is here that we part company with Juul, as it is our assertion that prioritizing one aspect of video gaming and video game culture, such as the act of play, in the words of Newman (2004: 153) 'impoverishes the study of video games'. Certainly, Juul's argument can be challenged on two key fronts. First, as Carr (2006) argues, just because an event happens simultaneously with its viewing by an audience - where story time and reading/viewing time occur together, to use Juul's terminology — does not necessarily mean there is not a story or narrative here. For example, and very relevant to our case here, Carr (2006: 39) argues that sports (and we would add sports-themed video games too) have a 'simultaneous narrative', which unfolds as the game is played and viewed by the audience. Second, following Grodal (2003), it can be argued that all narratives are an embodied process; or to paraphrase Heideggerian phenomenology, to be embodied is to always-already be in meaning. That is to say, irrespective of whether events are unfolding in 'real time' or are historic, the narrative is created in and through 
the actions of the audience, whether that is comprehending Hamlet's situation, hating Voldermort, or physically kicking a ball.

It is the audience who strings together components (or 'signs' to use the language of semiotics), such as words or images, to construct a coherent and logical narrative; it is the audience that projects meaning upon any and all experiences. And as communication studies tells us, the story that the recipient of a message/narrative constructs may be very different to that which the sender intended, or is held in their own mind (Longhurst et al., 2016). Narratives are never pre-existing or set, but rather are continuously made and re-made. Hence, it is our argument that simply because a video game may not appear to have a traditional pre-existing story, this does not necessarily mean it is devoid of narrative, or will not be constructed into a narrative(s) by those who play them, watch others playing them, or hear the tales about the playing of them.

In particular, a consideration and focus on experience allows us to see that Murray (1997) and Juul's (2001) respective positions and arguments are not as different as many may have first assumed. As, it appears that as with Murray, Juul also sees the playing of video games as an experience, which the gamers constructs using the components the game presents to them. As Juul (2001: online) writes: 'I would like to state that I think we need to consider games as fairly formal structures that in complex ways spawn and feed player experiences'. The only real difference is that Juul emphasises the importance of the ludic aspects of the game, while Murray focuses most of her attention of the narrative elements. However, one could easily argue - as we wish to here - that this kind of binary is disingenuous: to play is to construct meaning, and to construct meaning across the time of play is to build a narrative. This holds true whether one is playing Heavy Rain, Minecraft, or FIFA '17, as any Let's Play Youtube video proves.

Hence, it can be argued that even video games that do not have a traditional and pre-existing in-game narrative can still be experienced (and recounted) by the gamer as a narrative experience. For example, video gamers often talk in terms of what happened and what they or others did or felt when they were playing a video game. This includes, indistinctively on most occasions, the events happening in the gameworld and also what is happening in the space they are physically occupying in that moment. For example, this is explored by AUTHOR1 (2006) in his consideration of the players of 
Championship Manager and Football Manager, where conversations about in-game experiences easily and often blur with discussions of events in the world of professional association football. AUTHOR1 (2006: 526) argues that constructing an in-game experience that heavily refers to the out-of-game world of professional football 'allows the game to be drawn on [by the gamers] as a resource and narrative beyond conversations relating specifically to these games, and in particular, will often cross-cut and merge with those relating to football'.

\section{Enacting Experience}

Our fourth theme is how video games are enacted and embodied experiences. Costello (2016) defines video games as a kind of rhythmic experience. According to her, if the player wants to play a game, they 'must open their attention to the rhythms of the game and bend their behaviour to synchronize with the rhythms of action and response that the game requires' (Costello, 2016: 4). In this sense, playing a video game involves an embodied choreography between the game and the player; some of which will 'require precise rhythmic performance' (Costello, 2016: 4). Following the rhythm metaphor, the interactive experience of video gaming could be described thus as a rhythmic groove that the player 'may feel in or out of sync with and in or out of control of' (Costello, 2016: 5). Borrowing Heidegger's notion of 'attunement', musical connotation intact, James Ash (2013: 46) suggests similarly that video game players 'actively attune themselves to these environments, "mattering" new relations between the body-brain-environment assemblage as they go... that crosses both affective and emotional levels'.

This understanding of the gaming experience as deeply embodied and rhythmic could be related to Csikszentmihalyi's (1988) concept of 'flow', or certainly how it has been applied by several authors, such as by AUTHOR3 (2010) to the experience of playing Pro Evolution Soccer (PES). In particular, AUTHOR3 discusses how players of PES would communally work together to help maintain a 'flow' state; or to apply Costello's terminology, maintain the rhythms of the game experience. For example, AUTHOR3 discussed how the players he studied would mute the game commentary and instead play loud music, which helped keep them focused on the game and in a 'gamestate':

It seemed that the custom of playing music alongside the game would help the user reach the flow state in an accelerated time, and also would improve the 
duration, as the commentary (the Pro Evo series' commentary is renowned for being of a poor and often bizarre quality) would be muted, removing any distraction or the breaking of the suspension of disbelief through completely inaccurate reporting of the gamestate that sometimes occurred (AUTHOR3 2010: 340).

Csikszentmihalyi defines flow as a deep psychological state participants go into while engaging in a challenging activity; hence, flow is an internalised psychological state. This is why we find Costello's construct more useful, as it situates the experience of play as articulation and embodiment; a shared construction of rhythm between body and environment.

In particular, through the successful translation of meaning between domains, sports-themed video games allow players to enact, or at least play at, participating in sport at the highest level. This, AUTHOR1 (2008) argues, is one of the key attractions of playing a sports-themed videogame, and why they have consistently proved more popular than sports-themed films, certainly for many sports fans. Here, AUTHOR1 employs the work of Sandvoss (2003) and Hills (2002) on iconic and textual fandom. While iconic fandom, as the name would suggest, is about following a particular celebrity (an 'icon'), textual fandom describes fan affiliations to more complex ('textual') entities, such as, for example, a football club.

A football club, or another sports clubs for that matter, are never just one thing, but are rather a complex assemblage of the team, its players, manager, stadium, history, team colour, fans, and much more. This, Sandvoss argues, makes sports clubs polysemic; they are open to multiple, and often individual, readings. And, Sandvoss continues, this allows fans to see a reflection of themselves in the club that they follow. This is why fans of sports clubs use the pronoun of 'we' (such as, 'we won on Saturday') when describing 'their' team; as they feel part of the club or team they follow. Sports fans feel involved in the clubs they follow, in fact, they are not just followers, they are supporters: they feel connected as part of the club's architecture. It is through their loyalty, their purchasing of match tickets and merchandise, singing or shouting at games, or even wearing their lucky socks or pants, that they contribute to the team's success. In fact, these latter examples have themselves been turned into a app based videogame called Non-League Football Support, where the player of the game gets to simply choose what combination of lucky clothing, food, drink and who they 
take with them to each match, and then see the outcome; with little or no idea if the combination they chose had an effect on the result.

Hence, this, AUTHOR1 (2008) suggest, helps explain the popularity of sportsthemed video games, and the relative failure of cinema to make successful films about sports clubs or teams. Because, when a fan is watching a film focusing on a sports team, their lack of participation is starkly clear, and this makes the film distinctly different to their typical sports fandom experience where they, literally, feel incorporated. In this sense, sports-themed video games play into the sense of involvement prevalent at live sporting events or indeed even via television broadcast: the team colours, the screams and cheers, the feeling connected to other fans and the athletes upon the pitch. Just as being a part of the club they follow, the sports-themed videogame allows them to enact the experience of active participation in the team's success (or failure) to a hyperreal degree: controlling the squad roster, player development, team tactics, et cetera.

This, we would suggest (as highlighted above), is why the default perspective in the vast majority of sports-themed video games is the televisual gaze and not that of the player or manager. The player sees the game from the perspective of the sports-fan; from the perspective of a television camera. The sports-themed videogame simulates the fan's experience, but seeks to enhance this through the perception of even greater levels of control and involvement in the experience. Importantly, this then also relates back to some of our previous arguments: how video games are increasingly becoming a repository of technologically mediated experiences, and how this is one example of a wider trend of selling the consumer a sense of experience, which is often both new and familiar to them.

Video games are, therefore, amenable to translating the meaning of sport across domains because they must be enacted; an enactment that involves the player(s), the videogame, the screen, other people, the space in which the action is taking place, the controllers, the furniture, the lighting, the sound, the Internet connection, other devices, potential disruptions, past experiences, manuals, guides, conversations, and so forth. Playing a videogame is an enacted experience that involves a setting, a script, and a handful of colourful and diverse actors.

As with the world of sport, the world of video games too makes sometimes excessive demands upon one's body; as Westecott (2008: 11) puts it, our 'physical body is always implied in the game', one way or another. Furthermore, we would state that 
the presence of the body is more of an explicit matter than implicit. Once again, AUTHOR3's work on Pro Evolution Soccer gamers is illustrative here:

The body language of participants during play sessions was extremely distinctive depending upon the member's role. For example, a seated spectator would normally sit relaxed, backwards on the furniture, eyes wandering around the room at regular junctures. For those actively playing this posture was reversed; leaning very much forwards towards the screen, the entire upper body tensed with the eyes completely focused upon the television. The game controllers were also held in a distinctly forward manner, as the user consistently seemed to hold their pad as far in front of their body as comfortably possible, resting their elbows upon their thighs with the gamepad the closest object to the visual display (AUTHOR3, 2010: 351-352).

Hence, we would argue that video gaming is, again like sport, an explicit, insistent experience of bodily participation; of literal in-corpor-ation, as one picks up the controller (or clicks on the mouse), and feels embodiment with and in the world of sport. It is the video gamer, its bodily, material, and fleshy presence, which makes, in association with other material and semiotic elements, the gaming experience possible. Certainly, video games could be part of what Dourish (2001: 102) theorizes as embodied interaction, which is not just a form of interaction that is embodied, but an 'approach to the design and analysis of interaction that takes embodiment to be central to, even constitutive of, the whole phenomenon'. After all, as Dourish claims, embodiment is 'a property of interaction' (2001: 189) and not just systems, technologies, or artefacts (or even bodies, we should add). Video games can definitely be understood as 'a paradigmatic site for producing, imagining, and testing different kinds of relations between the body and technology in contemporary culture' (Lahti, 2003: 158).

\section{Conclusions}

In this paper, we have explored the idea of video games as designed experience, and more specifically, as a translated, narrated, and explicitly embodied experience, mediated by some sort of interactive digital technology. This leads us to recognize four key ways in which video games can be associated with experience, and how this helps us to understand the nuances of the gaming experience.

To begin with, video games connect with, but also lead, a wider social trend: understanding social reality as a set of designed experiences. The "real" is progressively becoming a repository of technologically mediated experiences and the logic of video 
games is anticipating this process. Second, video games can be seen as translations of meaning between domains. Developers often make efforts to research particular topics in order to translate the meaning of others' personal or social experiences into a game experience. Video games are, in this sense, technologically mediated experiences of other experiences; meta-experiences, structuring structures (Bourdieu, 1989). Third, we visualize video games as experiences because frequently social actors narrate their encounters with video games as they would other experiences such as a trip, an anecdote, a party, a problem at home or work, and so forth. They are part of the experience pool of everyday life. Fourth, video games are explicit bodily experiences since they insist upon the users' enactment in order to exist. This enactment requires a diverse set of actors who are demanded to perform a social choreography to bring the experience into being. In sum, video games are curated, designed, and enacted experiences of experiences, which we would suggest reflects a wider trend in not only videogame design but also the larger production and consumption techniques taking hold within an experience economy.

Finally, we would suggest that this paper therefore makes three key and significant contributions to knowledge. First, it operationalizes and defines the primary ways in which writers on gaming typically use the term 'experience'. As we have argued throughout this paper, experience is probably one of the most commonly associated terms with both video game play and games design. However, for a term so commonly used and associated with video games, in both their design and play, it is a concept few have taken the time to explicitly define or explain at any length. Hence, this paper sets out a clear framework for understanding how this term is, has been, and can be applied to video games.

Second, this paper frames video games within a social and economic context and suggests that in many respects contemporary video games provide a key exemplar of a wider social process, which increasingly sees social reality outlined as a series of designed experiences. It is suggested that there has been a social and economic shift towards selling and consuming experiences and that video games are at the vanguard of this. Moreover, being one of the most popular contemporary media formats, it could be argued video games are this paradigm's key driver (see AUTHOR2 \& AUTHOR1, 2018). 
Third, we suggest that experience actually offers a common ground in the (now dated, but still relevant) 'narratology versus ludology' debate. For both 'sides' of this argument experience is a key concept in understanding how games are designed, played, and engaged with. It seems that writers such as Murray (1998) and Juul (2001) are at odds due to focusing their attention on certain facets of the gaming experience. But we would suggest that by taking a more holistic approach, and understanding the various aspects and meanings of the gaming experience, we can maybe finally put to rest old and partial perspectives that only focus their lens on particular aspects of what it means to experience a video game.

\section{References}

Aarseth, Espen (2007). “Allegories of Space: The Question of Spatiality in Computer Games", in F. von Borries, S.P. Walz \& M. Böttger (eds), Space Time Play: Computer Games, Architecture and Urbanism: The Next Level, Berlin: BirkHäuser, pp. 44-47.

Adams, Ernest (2010). Fundamentals of Game Design (2 ${ }^{\text {nd }}$ Edition). Berkley, CA: New Riders.

Adi (2011). "The Death of Championship Manager - as I saw it", Sportskeeda, https://www.sportskeeda.com/football/the-death-of-championship-manager-as-isaw-it [Last Accessed: 21/07/2017].

Albrechtslund, A.M (2010) "Gamers Telling Stories: understanding narrative practices in an online community", Convergence, 16 (1): 112-124.

Ash, James (2013). "Technologies of Captivation: Videogames and the Attunement of Affect.", Body \& Society 1, 19: 27-51.

Bayliss, Peter Eric (2008). "Playing for Keeps: A Game of Marbles and the Materiality of Gameplay - Peter Eric Bayliss - Refractory: A Journal of Entertainment Media." Refractory: A Journal of Entertainment Media 13: Online.

http://refractory.unimelb.edu.au/2008/05/13/playing-for-keeps-a-game-of-marblesand-the-materiality-of-gameplay-peter-eric-bayliss/.

Baudrillard, Jean (1994). Simulacra and Simulation. Ann Arbor: The University of Michigan Press.

Bogost, Ian (2007). Persuasive Games. Cambridge, MA: MIT Press 
Bogost, Ian (2013). “What are Sports Videogames?”. In Consalvo, M., Mitgutsch, K. \& Stein, A. (eds). Sports Videogames. London: Routledge, pp. 50-66.

Bryman, Bolter, Jay David, and Richard Grusin (1999). Remediation: Understanding New Media. Cambridge: MIT Press.

Bourdieu, Pierre (1989). "Social space \& symbolic power", Sociological Theory, 7: 1425.

Carr, Diane (2006). “Games \& Narrative”. In D. Carr, D. Buckingham, A. Burn, \& G. Schott (Eds.). Computer games: Text, narrative and play. Cambridge: Polity Press, pp. 30-44.

Chatman, Seymour (1978). Story and Discourse. Ithaca, NY: Cornell University Press.

Costello, Brigid Mary (2016). "The Rhythm of Game Interactions: Player Experience and Rhythm in Minecraft and Don't Starve", Games and Culture, doi: $10.1177 / 1555412016646668$.

Csikszentmihalyi Mihaly. (1988) Optimal Experience: psychological studies of flow in consciousness. Cambridge: Cambridge University Press.

Dourish, Paul (2001). Where the Action Is. The Foundations of Embodied Interaction. Cambridge, Massachusetts: MIT Press.

Grodal, Torben (2003). "Stories for Eye, Ear, and Muscles: Video Games, Media, and Embodied Experiences" in Wolf, Mark J. P. and Perron, Bernard. The Video Game Theory Reader. London: Routledge, 129-155.

Hill, Charles (2004). "The Psychology of Rhetorical Images.” In Marguerite Helmers and Charles A. Hill (editors). Defining Visual Rhetorics. Mahwah, N.J.: Lawrence Erlbaum Associates, 25-40.

Hills, Matt (2002). Fan Cultures. London: Taylor \& Francis.

Hutchins, Brett (2008). "Signs of meta-change in second modernity: the growth of esport and the World Cyber Games", New Media and Society, 10 (6): 851-869.

Juul, Jesper (2001). “Games Telling Stories?”, Game Studies, 1 (1).

Kücklich, J (2006). "Literary Theory and Digital Games". In Rutter and Bryce. Understanding Digital Games. London: SAGE, pp. 95-111.

Lahti, Martii (2003). “As We Become Machines: Corporealized Pleasures in Video Games". In Wolf, Mark J.P. and Perron, Bernard (eds.). The Video Game Theory Reader. London: Routledge.

Longhurst, Brian; Smith, Greg; Bagnall, Gaynor; Crawford, Garry; Ogborn, Miles (2016). Introducing Cultural Studies (Third edition). London: Routledge. 
MacCannell, Dean (1973). "Staged Authenticity: Arrangements of Social Space in Tourist Settings", American Journal of Sociology, 73 (3): 589-603.

Murray, Janet H. (1997). Hamlet on the Holodeck: The Future of Narrative in Cyberspace. New York, NY: The Free Press.

Newman, James (2004). Videogames. London: Routledge.

Osborn, George (2016). "Football Manager 2017 review - the best in the series, but only for dedicated fans", The Guardian, https://www.theguardian.com/technology/2016/nov/02/football-manager-2017review-the-best-in-the-series-but-only-for-dedicated-fans [Last Accessed: 21/07/2017].

Pine, Joseph and Gilmore, James H. (2011). The Experience Economy. Boston: Harvard Bussiness Review Press

Popper, Daniel (2017). “Giants' Damon Harrison preparing for Green Bay by playing with Packers on Madden NFL", New York Daily News, http://www.nydailynews.com/sports/football/giants/giants-damon-harrisonplaying-madden-nfl-prepare-packers-article-1.2936426 [Last Accessed: 21/07/2017].

Salen, Katie and Zimmerman, Eric (2004). Rules of Play: Game Design Fundamentals. Cambridge, MA: MIT Press.

Sandvoss, Cornel (2003). A Game of Two Halves: Football, Television, and Globalisation. London: Psychology Press.

Shaw, Adrienne (2014). Gaming at the Edge. Sexuality and Gender at the Margins of Gamer Culture. Minneapolis: University of Minnesota Press.

Stallabrass, Julian. (1996). Gargantua: manufactured mass culture. London: Verso.

Stein, Abe (n.d.). "Madden turns 25 this year. 'It's time for it to start acting its age", Kill Screen, https://killscreen.com/articles/madden-turns-25-year-its-time-it-start-actingits-age/ [Last Accessed: 21/07/2017].

Stein, Abe (2013). "Playing the Game on Television", in M. Consalvo, K. Mitgutsch \& A. Stein (eds). Sports Video Games. London: Routledge, pp.: 115-137.

Stuart, Keith (2014). "Why clubs are using Football Manager as a real-life scouting tool", The Guardian, https://www.theguardian.com/technology/2014/aug/12/why-clubsfootball-manager-scouting-tool [Last Accessed: 21/07/2017].

Suits, B (2005). The Grasshopper: Games, Life and Utopia. Ontario: Broadview Press.

Taylor, T. L. (2009). Play between Worlds. Exploring Online Game Culture. Cambridge, MA: MIT Press. 
Taylor, T. L. (2012). Raising the Stakes. E-Sports and the Professionalization of Computer Gaming. Cambridge, MA: MIT Press.Westecott, Emma (2008). "Bringing the Body back into Play", The [player] conference proceedings, Copenhagen.

Witkowski, Emma (2012). "On the Digital Playing Field. How We "Do Sport" With Networked Computer Games", Games and Culture, 7 (5): 349-374. 